\title{
Archéopages Archéopages
}

Archéologie et société

45 | 2017

De la terre au pot

\section{L'Argonne et la question des sigillées tardives en Île-de-France}

De nouvelles perspectives

The Argonne and the question of late samian ware in Île-de-France. New perspectives

Argonne y la cuestión de las sigillata tardías en Isla de Francia. Nuevas

perspectivas

\section{Philippe Bet, Rémy Guadagnin et Renaud Gosselin}

\section{OpenEdition}

\section{Journals}

\section{Édition électronique}

URL : https://journals.openedition.org/archeopages/2932

DOI : 10.4000/archeopages.2932

ISSN : 2269-9872

Éditeur

INRAP - Institut national de recherches archéologiques préventives

Édition imprimée

Date de publication : 1 mars 2018

ISSN : $1622-8545$

\section{Référence électronique}

Philippe Bet, Rémy Guadagnin et Renaud Gosselin, « L'Argonne et la question des sigillées tardives en Île-de-France », Archéopages [En ligne], 45 | 2017, mis en ligne le 24 juillet 2018, consulté le 03 juin 2021. URL : http://journals.openedition.org/archeopages/2932 ; DOI : https://doi.org/10.4000/ archeopages.2932 


\section{L'Argonne et la question des sigillées tardives en Île-de-France De nouvelles perspectives}

Philippe Bet Inrap, UMR 5138, «Archéologie et Archéométrie»"

Rémy Guadagnin

Renaud Gosselin

56

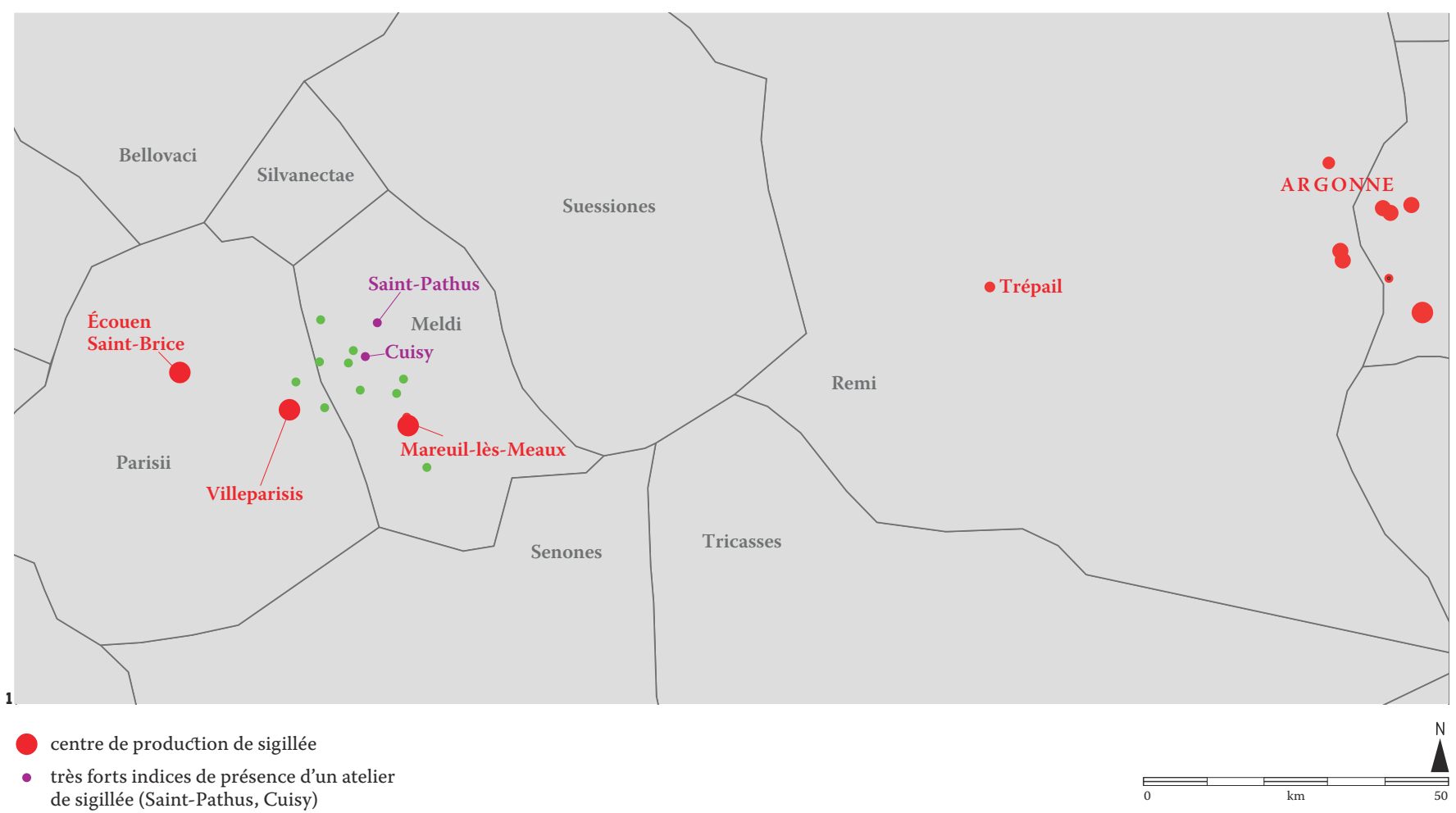

- artefacts en relation avec la fabrication de sigillée

- limites supposées des cités romaines 
L'Argonne est une région de production de céramiques bien connue pour l'Antiquité romaine (Mitard et al., 1986 ; Gazenbeek, Van der Leeuw, 2003). Sur près de $200 \mathrm{~km}^{2}$ (Brulet, Feller, 1997 , 2003), de nombreux ateliers de potiers ont fabriqué, dès le $\mathrm{I}^{\mathrm{er}}$ siècle, de la céramique fine de type terra nigra et des céramiques communes. À partir du $\mathrm{II}^{\mathrm{e}}$ siècle, ils se mirent à produire de la sigillée (Chenet, Gaudron, 1955), mais également des parois fines engobées et des métallescentes. Pour la céramique sigillée, les officines employèrent une argile verte non calcaire. En cela, elles différaient des grands centres de production comme Arezzo, dans la seconde moitié du I ${ }^{\mathrm{er}}$ siècle avant notre ère, ou Millau, à partir des années 10 ou 15, qui, avec des argiles calcaires à plus de $7 \%$, pouvaient cuire leurs produits à plus de $1000{ }^{\circ} \mathrm{C}$ et obtenir des vernis brillants et bien étanches. Dès le tout début du $\mathrm{II}^{\mathrm{e}}$ siècle ou quelques années auparavant, la fabrication de sigillées à pâte calcaire se propage malgré la complexité technique de sa réalisation, et de multiples ateliers font leur apparition. Mais durant cette même période, les artisans argonnais, dont certains venant de Gaule centrale, fabriquent une sigillée à pâte toujours siliceuse. Ils restent fidèles à l'emploi d'une telle argile jusqu'à l'arrêt de cette fabrication, sans doute à l'aube du vi ${ }^{\mathrm{e}}$ siècle [ill. 1]. Celle-ci, en effet, n'aurait pas pu supporter, sans se déformer, une cuisson aux alentours de $1000^{\circ} \mathrm{C}$ et l'obtention du grésage qui aurait conféré aux vernis, par la vitrification, une dureté et une imperméabilité absolues.

Comme leurs homologues de Lezoux ${ }^{\mathbf{1}}$ durant le $\mathrm{I}^{\mathrm{er}}$ siècle, les potiers ont adopté pour la cuisson des sigillées des fours à tubulures. Ils cuisaient leur production par rayonnement sans l'exposer au contact direct des flammes. Cette technique nécessitait une montée en température du four plus forte en raison d'une importante déperdition de la chaleur. D'autres potiers auraient pu, dès le début de la phase de refroidissement, faire un apport massif d'air et, grâce à cette arrivée d'oxygène, obtenir un état de surface assez proche de l'effet recherché, mais peut-être plus incertain. Finalement, les sigillées argonnaises offrent un engobe orangé régulier, parfois presque brillant, et une pâte orange d'une grande homogénéité.

Dans le quart de siècle qui succède aux décennies troubles de la fin du $\mathrm{III}^{\mathrm{e}}$ siècle, les potiers d'Argonne fabriquent de la sigillée avec un répertoire particulier. Même si ce dernier emprunte encore au répertoire en vigueur auparavant, l'évolution de certaines formes est si marquée qu'elles en deviennent originales. L'une d'entre elles allait devenir emblématique : la coupe ornée appelée Chenet 320 (Chenet, 1941). Jusqu'alors, les potiers argonnais proposaient, comme les autres centres de production sigillée, des coupes moulées hémisphériques (Drag. 37). Les potiers argonnais avaient, par ailleurs, conservé dans leur répertoire une forme ancienne qui avait été inventée à Lezoux (Loo8) à la période flavienne et fabriquée dans le grand centre arverne jusque dans la première moitié $\mathrm{du} \mathrm{II}^{\mathrm{e}}$ siècle. Elle reprenait la forme hémisphérique du Drag. 37, mais son décor moulé était remplacé par un guillochis réalisé à la lame vibrante sur le tour. Le génie des artisans d'Argonne a été, en ce début $\mathrm{du} \mathrm{IV}^{\mathrm{e}}$ siècle, de remplacer le décor moulé ou guilloché par une série de motifs obtenus avec une molette appliquée de façon concentrique sur la panse du vase. La réalisation était plus rapide qu'avec un moule et le décor plus complexe et varié qu'un guillochis. La molette (Feller, 1991) était composée d'une série de casiers avec des motifs essentiellement géométriques (hachures, croix, points, oves...). Dans la seconde moitié du $\mathrm{V}^{\mathrm{e}}$ siècle, des motifs chrétiens (poisson, chrisme, homme allongé) apparurent également sur ce type de vases (Bayard, 1990). L'ensemble de la production présentait une autre différence de taille par rapport à celle du siècle précédent, l'abandon total de l'estampillage, y compris pour les plats et les assiettes. Cela peut paraître antinomique pour une céramique qualifiée de « sigillée », mais elle s'inscrit pourtant bien dans l'évolution de cette catégorie.

Pendant très longtemps, dès qu'un vase en sigillée décoré à la molette était découvert, on lui attribuait l'Argonne comme origine. À la fin du $\mathrm{XX}^{\mathrm{e}}$ siècle, trois chercheurs (Bakker et al., 1996) s'attelèrent au travail ardu d'inventorier les molettes employées par ces officines argonnaises ${ }^{2}$. L'un d'eux, Paul Van Ossel (Van Ossel, 2011a), s'aperçut que la situation devait être plus complexe. Un certain nombre de molettes ne se retrouvaient que dans certains secteurs de l'îlede-France et les vases sur lesquels elles étaient appliquées avaient une pâte moins orangée que celle de l'Argonne. L'hypothèse d'une nouvelle région de production était alors émise, mais la question de sa localisation demeurait ouverte.

\section{La villa de Mareuil-lès-Meaux et son officine de sigillée}

La réponse à cette question a été apportée par l'archéologie préventive. Au tout début des années 200o, un important diagnostic archéologique ${ }^{3}$ a été prescrit sur l'emprise du contournement routier de Meaux. Il a mené à la découverte, sur la commune de Mareuil-lès-Meaux, dans la pars rustica d'une villa, d'un four de potiers, avec des tubulures encore en place. Des tessons de sigillée, notamment certains avec un décor à la molette NS1099 défini par Paul Van Ossel, étaient associés à ces vestiges. La fouille de la villa a été effectuée en 2002 (Bet, 2003 ; Bet et al., 2003, $2008)^{\mathbf{4}}$. Elle a mis au jour un atelier de potiers du $\mathrm{IV}^{\mathrm{e}}$ siècle, dans la partie sud-est de l'établissement. Quatre autres fours de potiers ont été dégagés, ainsi que d'importants remblais-dépotoirs totalisant 11 ooo céramiques et terres cuites et des centaines de kilos d'éléments de four [ill. 2]. Le degré d'arasement du site n'a pas permis la mise au jour 


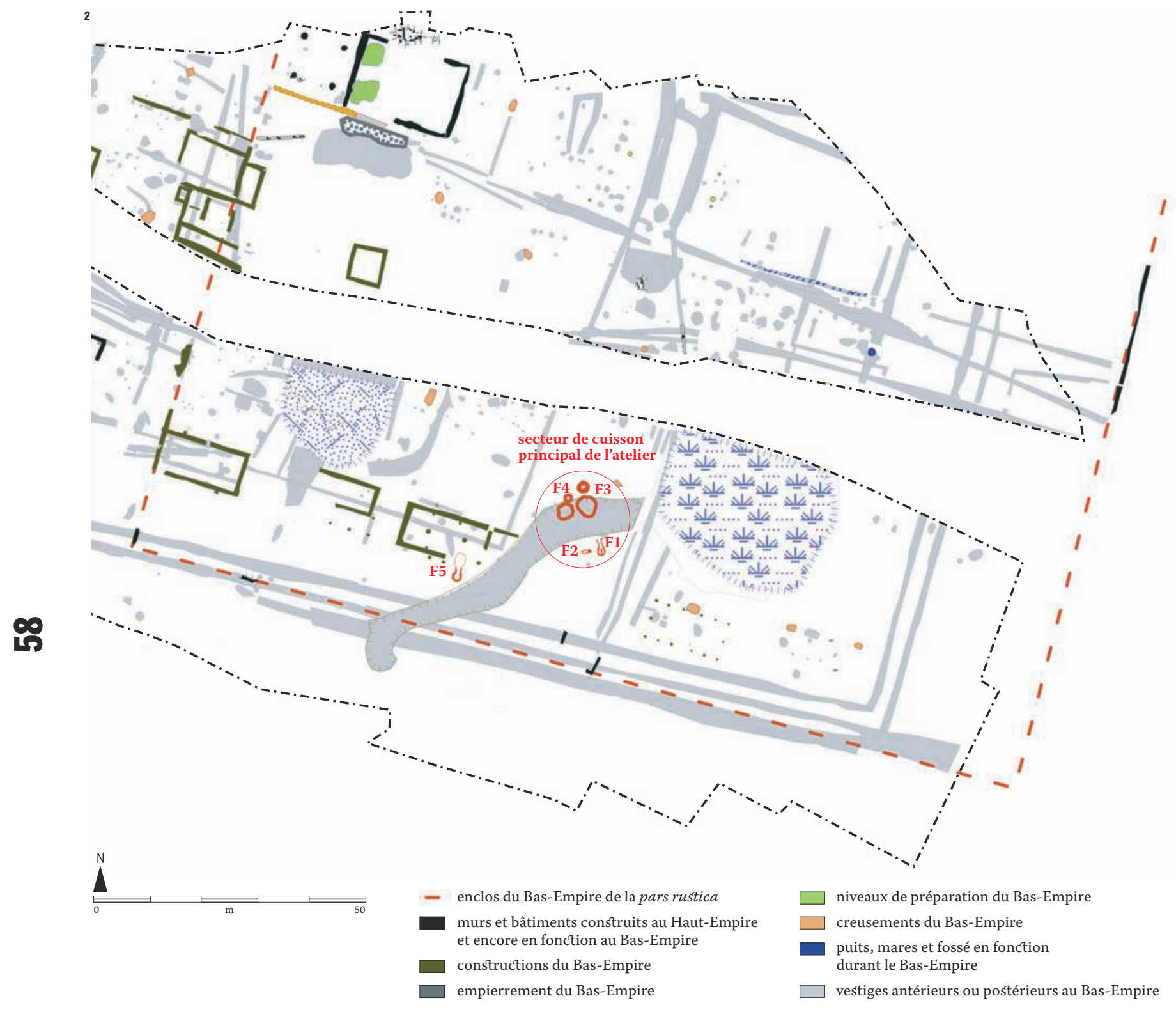

3. $a$

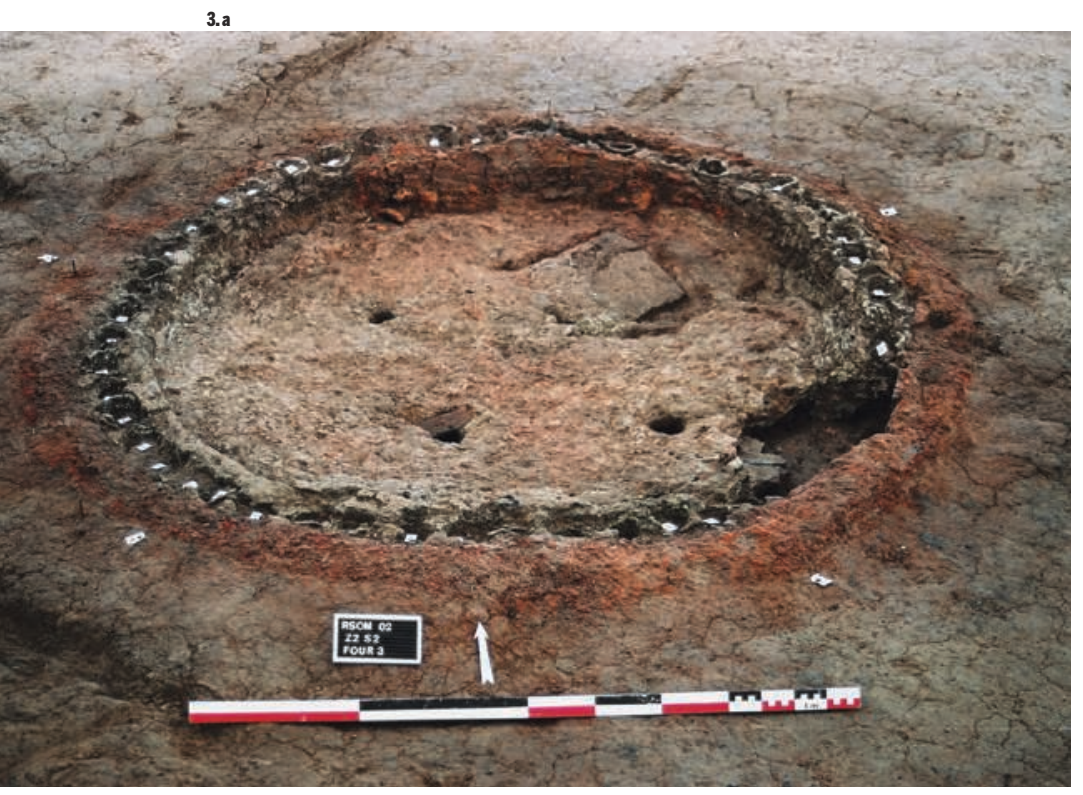

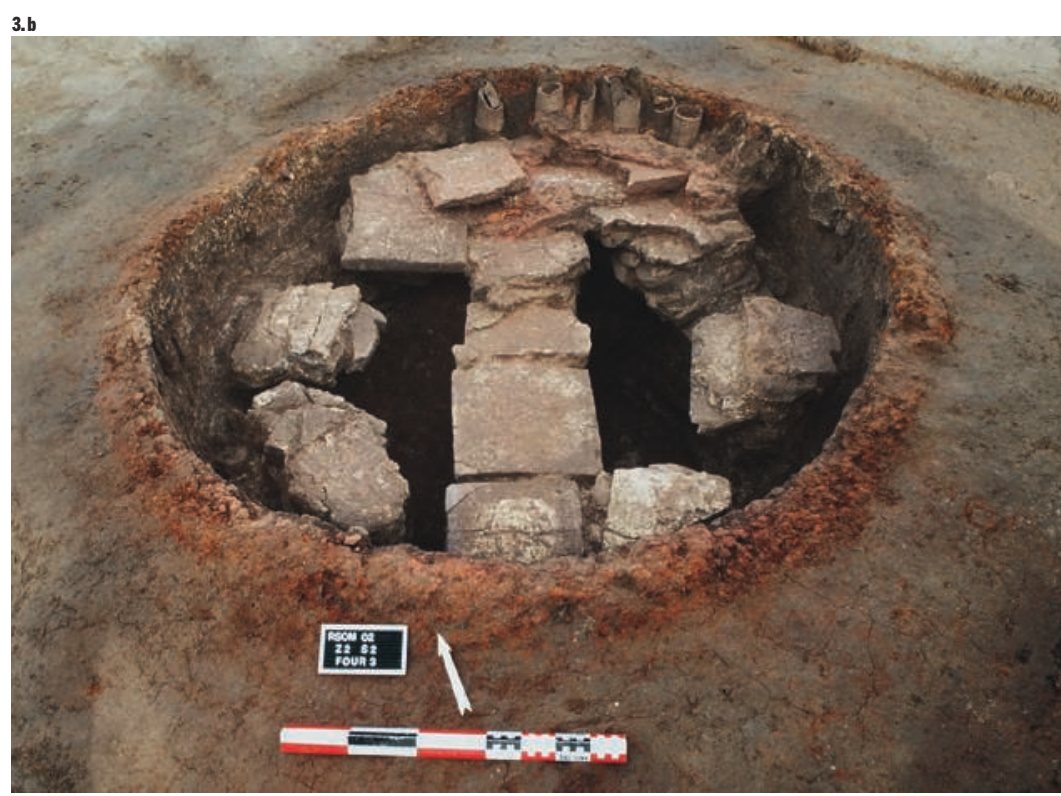


2. Plan de la pars rustica de la villa de Mareuil-lèsMeaux et des fours de potiers du Bas-Empire.

3. a. Le four à sigillée $n^{\circ} 3$

de Mareuil-lès-Meaux avec sa sole percée de quatre carneaux et ses tubulures périphériques en place. b. Le four en cours de démontage: les supports de sole et quelques tubulures. de certaines structures liées à l'activité potière (tours, aire de préparation de l'argile...). L'un des fours avait été construit dans une annexe, d'une superficie de $18 \mathrm{o} \mathrm{m}^{2}$, de la villa du Haut-Empire. Seuls l'alandier et la salle de chauffe étaient inclus dans l'ancien bâtiment, ce qui permettait l'alimentation en bois et la conduite de la cuisson dans un espace couvert. À $15 \mathrm{~m}$ de là, quatre autres fours étaient installés dans une ancienne dépression. Celle-ci, n'étant comblée que partiellement à cette époque, a dû servir de salle de chauffe pour alimenter les alandiers en combustible. Deux de ces fours étaient munis de tubulures pour la cuisson de la sigillée, un autre était destiné à la cuisson de poteries plus ordinaires et le quatrième était trop détruit pour déterminer sa fonction. Dans un premier temps, un premier four à sigillée a été construit dans la partie sud de la dépression. À la suite, sans doute, d'un accident de cuisson, ce premier four a été abandonné. Un nouveau four a alors été bâti, de manière très similaire, à quelques mètres du précédent. Dans la salle de chauffe, plusieurs niveaux de sol et de remblais, composés de déchets de cuisson, semblent indiquer une période d'utilisation plus longue que pour le four précédent [ill. 3a et 3b]. Après l'abandon du second four à sigillée puis du petit four qui était à côté, toute la dépression a été remblayée avec les tessonnières qui devaient s'élever tout autour. Le fonctionnement de cet atelier n'a pas dû dépasser une trentaine d'années. Quelques monnaies retrouvées dans les couches liées à ces fours témoignent d'une activité concentrée sur le troisième quart du IV ${ }^{\mathrm{e}}$ siècle et confortent les datations indiquées par les céramiques.

Plusieurs indices montrent que les potiers de Mareuil-lès-Meaux viennent très probablement d'Argonne et qu'ils ont, sans doute, voulu installer une unité de production à proximité immédiate de la capitale de cité des Meldes. Ils savaient parfaitement tourner et décorer leurs vases en respectant scrupuleusement les normes morphologiques et typologiques établies dans les officines argonnaises. Ils ont ainsi produit des coupes hémisphériques de différents modules, de grands plats et des assiettes, des mortiers à râpe interne, des bols ou des coupelles, des gobelets et des cruches [ill. 4]. Les décors utilisés sont variés : peints, excisés, imprimés en creux à l'aide d'une molette. Ces derniers peuvent être classés en une dizaine de types différents dont deux seulement sont couramment mis en œuvre par les potiers de Mareuil. Certains accessoires de cuisson portent des graffites IIV similaires à ceux retrouvés en Argonne sur les mêmes supports. Les artisans ont fabriqué différents types de tubulures quasiment identiques à ceux des grands ateliers argonnais, mais ils ont moins bien maîtrisé la fabrication d'un modèle de tubulure qu'ils ont trop étiré [ill. 5]. Cela a provoqué des défaillances dans l'étanchéité du four vis-à-vis des fumées, celle-ci devant être parfaite pour la sigillée. Le vernis rouge orangé des vases s'en est trouvé altéré et, comme ils n'ont pas pu cuire ou refroidir dans une atmosphère uniquement oxydante, les pâtes présentent, parfois, un cœur gris ou noir.

Les potiers de Mareuil ont prélevé de l'argile directement sur les pentes du plateau où ils se sont installés. Ils ont principalement employé une argile verte non calcaire, avec des nodules carbonatés, qu'ils ont cuite aux alentours de $900{ }^{\circ} \mathrm{C}$ (Bet et al., 2011 ; Delbey et al., 2013). Beaucoup plus étrangement, ils ne sont pas contentés d'utiliser cette argile proche de celle que l'on trouve habituellement en Argonne mais offre une couleur de pâte moins orangée et plus brune après cuisson. En effet, ils ont extrait, à proximité immédiate du premier gisement, de l'argile blanchâtre et riche en calcaire (12\%). Ils ont été contraints de pousser davantage leur four pour atteindre une température de cuisson située entre $975^{\circ} \mathrm{C}$ et $1039^{\circ} \mathrm{C}$ ce qui peut être considéré comme une véritable prouesse avec un four mouflé. Cela implique également que les potiers de Mareuil ont réalisé des chargements complets de plusieurs centaines de vases obtenus avec ce type d'argile. L'emploi d'une argile calcaire et l'obtention d'une température aussi élevée n'ont jamais été constatés en Argonne. Cela démontre une certaine adaptabilité des potiers de Mareuil et le fait qu'ils ne se sont pas contentés de répéter les schémas appris dans leur atelier d'origine. S'ils n'ont pas apporté de modification au répertoire typologique, ils ont, peut-être, essayé de renouer avec la longue tradition de la vraie sigillée à pâte calcaire héritée d'Arezzo. Cette tentative est cependant restée sans lendemain et ne correspond qu'à une part très faible de la production de l'atelier de Mareuil.

\section{D'autres ateliers de sigillée}

Si l'atelier de sigillée de Mareuil-lès-Meaux a été le premier à être découvert en Île-de-France, il n'est pas isolé (Bet, Delage, 2008 ; Barat 2011). Grâce à une association d'archéologues bénévoles, la Jeunesse Préhistorique et Géologique de France (JPGF), des prospections systématiques et intensives de qualité ont pu être menées en plaine de France ${ }^{5}$. Elles ont permis de retrouver des supports de cuisson ou des tubulures sur une dizaine de sites archéologiques régionaux. Sur certains, la densité est telle, comme à Saint-Pathus ou à Cuisy (Seine-et-Marne), que la présomption d'un atelier de sigillée dans ces secteurs est forte. À Villeparisis, près de l'église Saint-Martin dont le vocable indique souvent une occupation du lieu au Bas-Empire, des fouilles ${ }^{6}$ ont révélé un four pour la cuisson de poteries granuleuses (Mallet, Mahé, 2003), mais également des dépotoirs de céramique sigillée qui indiquent, sans l'ombre d'un doute, une production sur place de celle-ci (Van Ossel, 2011b).

Dans la vallée du Rosne, dans un secteur à cheval sur les territoires communaux d'Écouen 

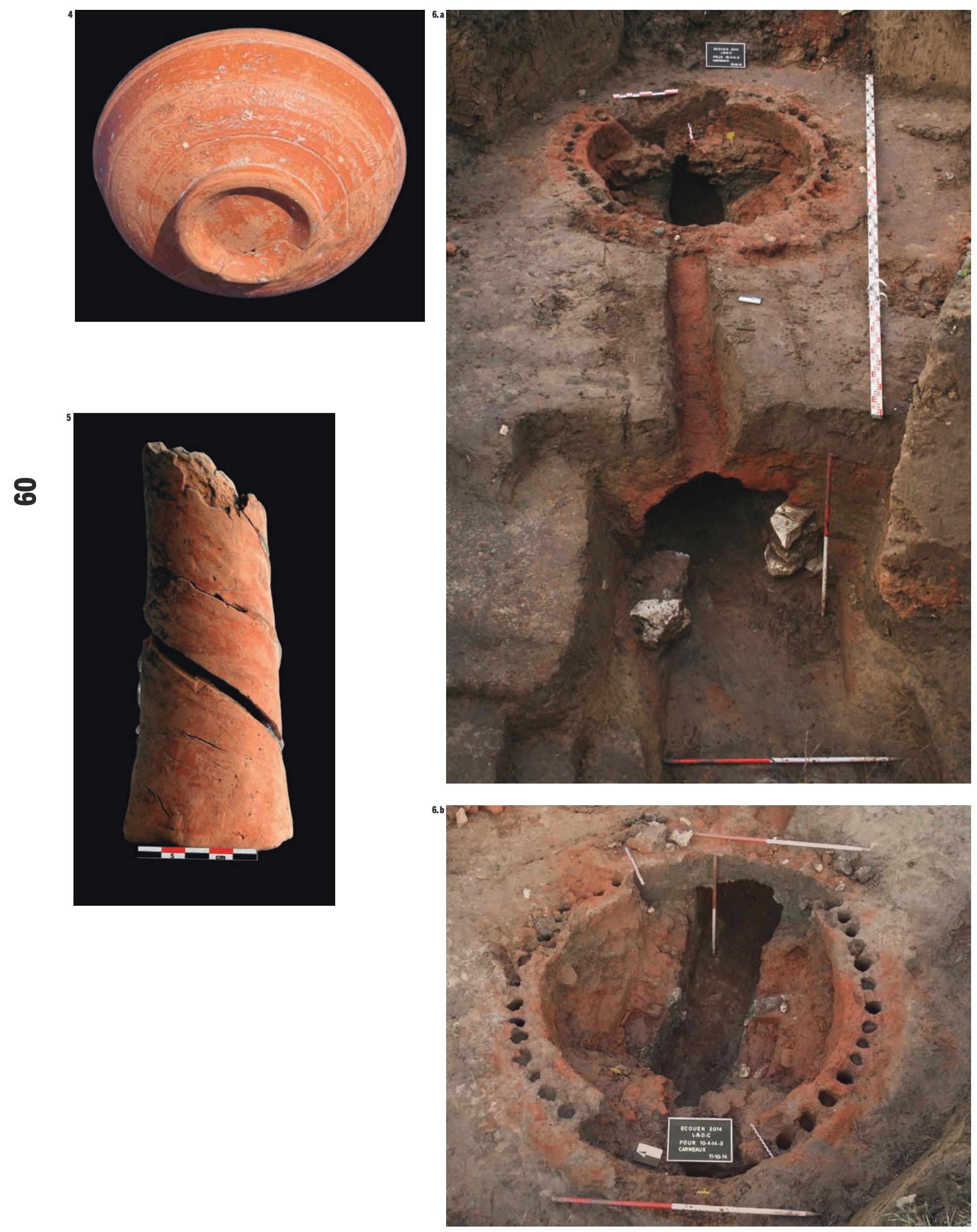


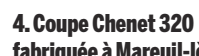

fabriquée à Mareuil-lès-

Meaux et décorée avec

la molette NS1099.

5. Tubulure présentant

un défaut de faconnage

de l'atelier de Mareuil-

lès-Meaux.

6. a. Le four à sigillée

de l'atelier d'Écouen/

Saint-Brice, avec son canal

de chauffe et sa couronne

de tubulures périphériques.

b. La couronne de tubulures

et les recharges en limon.

7. Menés sous

la responsabilité

de Rémy Guadagnin.

8. Fouille menée

de 2006 à 2007 sous

la responsabilité de

Franck Gama, Inrap. et de Saint-Brice (Val-d'Oise), la JPGF ${ }^{7}$ a réalisé, depuis quelques années, des sondages pour vérifier la présence d'un atelier de sigillée (Guadagnin, 2016). Les plus récents viennent tout juste de s'achever en septembre 2017. Ces travaux ont mis au jour quatre fours à sigillée du IV ${ }^{\mathrm{e}}$ siècle, implantés à proximité immédiate d'une villa galloromaine et en bordure de la voie antique reliant Lutèce à Samarobriva. L'un d'entre eux, assez bien conservé, est remarquable et unique dans le monde romain [ill. 6]. Sa partie inférieure a été littéralement taillée dans le limon argileux, sans utiliser de fragments de tegulae liées à l'argile pour consolider la construction. Le départ de chaque tubulure de la couronne périphérique du laboratoire est ainsi creusé dans la masse. L'alandier, de son côté, apparaît sous la forme d'un tunnel rectiligne long d'environ $2 \mathrm{~m}$ et formant un canal de chauffe d'une longueur totale de $4 \mathrm{~m}$. La paroi vitrifiée présente quelques recharges de limon en réparation [ill. 6b]. Les fours à sigillée comprennent souvent un alandier très allongé, sans doute pour mieux répartir la chaleur à son arrivée sous le laboratoire de cuisson. En bordure de la voirie, un poste de tournage a même été découvert. Des analyses sont actuellement en cours pour confirmer l'existence d'un deuxième : il convient de déterminer si, autour d'un calage d'axe fixe présumé, il s'agit bien d'argile verte du Sannoisien. Actuellement, sept molettes différentes sont clairement attribuables au site de production d'Écouen/Saint-Brice pour la décoration de coupes ornées Chenet 320.
En l'espace d'une quinzaine d'années, notre connaissance des ateliers de sigillée du Bas-Empire a considérablement évolué. Trois centres de production distincts, en territoires melde et parisien, offrent déjà une belle documentation et peuvent servir de référence. D'autres lieux ont fourni des indices sérieux pour y soupçonner l'existence d'un atelier. Pour la seconde moitié du IV ${ }^{e}$ siècle, la proportion de sigillée francilienne découverte dans certains sites archéologiques de la civitas des Meldes peut être relativement importante et avoisiner les $30 \%$. D’autres régions peuvent également être concernées par le déplacement de potiers argonnais. On connaît depuis peu le cas de Trépail, en territoire rème (Deru et al., 2003) qui se trouve à mi-chemin entre l'Argonne et Mareuil-lès-Meaux. Il faudrait également rechercher beaucoup plus à l'est de Verdun si des indices ne pourraient également indiquer, dans la vallée de la Moselle, des ateliers similaires. Cela ne serait pas étonnant puisque des officines de sigillée s'y étaient déjà implantées dans la seconde moitié du Haut-Empire.

La découverte de quelques ratés de cuisson et d'une tubulure lors de la fouille des abords du grand amphithéâtre de Metz ${ }^{\mathbf{8}}$, dans des contextes $\mathrm{du} \mathrm{v}^{\mathrm{e}}$ siècle, sera vraisemblablement à réexploiter. L'enquête sur les ateliers de sigillée de type argonnais n'en est, sans doute, qu’à ses débuts...
Références bibliographiques

Bakker L., Dijkman W., Van Ossel P., 1996, « Corpus de la céramique sigillée d'Argonne de l'Antiquité tardive », in Actes du congrès de Dijon, 16-19 mai 1996, Marseille, Société française d'étude de la céramique antique en Gaule (SFECAG), p. 423-426.

BARAT Y., 2011, « Imitations de sigillée argonnaise ou de céramique à l'éponge ? Céramiques fines à cuisson oxydante de l'Ouest parisien au Bas-Empire ", in VAN OSSEL et al., 2011, p. 255-262.

BAYARD D. 1990, « L'ensemble du grand amphithéâtre de Metz et la sigillée d'Argonne au v viècle », Gallia, t. 47, p. 271-319.

Bet P., Delage R., Van Ossel P., 2003, «Un atelier de sigillée de type argonnais près de Meaux, le site de la Grange-du-Mont à Mareuil-lès-Meaux (Seine-et-Marne) », in Actes du Congrès de SaintRomain-en-Gal, 29 mai-1 ${ }^{\text {er }}$ juin 2003, Marseille, SFECAG, p. 435-4.48.

Bet P., avec la coll. de Delage R., Van Ossel P., 2003, « Mareuil-lès-Meaux - Rocade sud/ouest de Meaux un atelier de céramique de type argonnais ", Actes des journées archéologiques d'Île-de-France, SRA, p. 113-130.

Bet P., Delage R., Gosselin R., Bojarski M., Benhaddou S., Charamond C., Maya-Pion M., Sethian E., 2008, Mareuil-lès-Meaux, La villa gallo-romaine et l'atelier de sigillée tardive de La Grange-du-Mont (77), rapport d'opération, InrapSRA Île-de-France, 4 vol., 1766 p.
Bet P., Delage R. 2008, « Les ateliers de céramique sigillée tardive », in GRIFfisch J.-N., MAGNAN D., Mordant D. (dir.), Carte Archéologique de la Gaule, 77/2 - La Seine-et-Marne, Paris, Académie des Inscriptions et Belles-Lettres, p. 198-203.

Bet P., Delage R., Picon M., Van Ossel P., Thirion V., 2011, « Un atelier de potier du IV ${ }^{\mathrm{e}} \mathrm{s}$. à Mareuil-lèsMeaux (Seine-et-Marne) », in VAN OSSEL et al., 2011, p. 337-366.

Brulet R., Feller M., 1997, « Recherches sur les ateliers de céramique gallo-romains en Argonne », Archaeologia Mosellana, 3, p. 1-140.

Brulet R., Feller M., 2003, « Recherches sur les ateliers de céramique gallo-romains en Argonne ", Archaeologia Mosellana, 5, p. 301-451.

Chenet G., Gaudron G., 1955, La céramique sigillée d'Argonne des II et III siècles, Gallia, VI, suppl. 6, $246 \mathrm{p}$.

CHENET G., 1941, La céramique d'Argonne du IVe siècle et la terre sigillée décorée à la molette, Mâcon, Protat frères, $195 \mathrm{p}$.

Delbey T., Guillon R., Van Ossel P., Petit C., GARCiA J.-P., 2013, « Caractérisations pétrographique et granulométrique d'imitations de sigillées d'Argonne ", in Actes du congrès d'Amiens, 9-12 mai 2013, Marseille, SFECAG, p. $465-474$.

Deru X., Redon B., Louvion C., 2003, " Prospections à Trépail, "Le Trou de la Baticaude" (Marne) : l'atelier de potiers et le site gallo-romain ", Revue du Nord, vol. 353, n 5, p. 181-208.

Feller M., 1991, «Classification et datation des molettes d'Argonne. Problèmes de méthodes ", in Actes du congrès de Cognac, 8-11 mai 1991, Marseille, SFECAG, p. 161-169.
Gazenbeek M., VAN der Leeuw S., 2003, « L'Argonne dans l'Antiquité : étude d'une région productrice de céramique et de verre ", Gallia, t. 6o, p. 269-317.

GuAdAGNIN R., 2016, «Écouen/Saint-Brice, les ateliers de potiers gallo-romains de la vallée du Rosne », Bulletin semestriel de la JPGF, $\mathrm{n}^{\circ}$ 1, p. 2-28.

Mallet F., MAhÉ N., 2003, « Villeparisis, "ZAC du Vieux Pays", un four de potier de céramique granuleuse du Bas-Empire ", Actes des journées archéologiques d'Île-de-France, SRA, p. 101-111.

Mitard H., Hofmann B., Lutz M., 1986, « Les ateliers de l'Est de la France. Groupe d'Argonne ", in BÉMONT C., JАСOB J.-P. (dir.), La terre sigillée gallo-romaine. Lieux de production du Haut-Empire : implantations, produits, relations, Paris, Éd. de la Maison des sciences de l'homme, « Documents d'archéologie française » 6, p. 196-207.

VAN Ossel P. (dir.) avec la coll. de Bertin P., SÉGUIER J.-M., 2011, Les céramiques de l'Antiquité tardive en Île-de-France et dans le Bassin parisien, vol. II, Synthèses, Nanterre, Diocesis Galliarum, « Document de travail » 9, 394 p

VAN OSSEL P., 2011a, « Les sigillées du groupe Argonne dans le Bassin parisien au Bas-Empire. Caractérisation, production et diffusion ", in VAN OSSEL et al., 2011, p. 231-254.

VAN OSSEL P., 2011b, « Les productions de céramique sigillée du groupe Argonne de Villeparisis », in VAN OSSEL et al., 2011, p. 321-336. 